\title{
The Role of Biomarkers in the Early Diagnosis of Endometriosis
}

\author{
Dominic Adam Worku* \\ Medical Doctor, University of Birmingham, UK \\ *Corresponding author: Dominic Adam Worku, Medical Doctor, University of Birmingham, UK \\ Submission: 进 October 17, 2017; Published: 㭗 November 14, 2017
}

\begin{abstract}
Endometriosis is a chronic gynecological problem which is frequent within the general population. Due to its varied presentations there is often a vast delay in diagnosis incurring a large amount of psychological and physical harm with infertility being unfortunate sequelae. Whilst improvements have been made with regards to the treatment of endometriosis, diagnosis remains challenging for clinicians with traditional investigations yielding poor results. This review will consider potential biomarkers and investigations for the early diagnosis of endometriosis.
\end{abstract}

\section{Introduction}

Endometriosis is an oestrogen dependent debilitating gynaecological condition that is underdiagnosed in women and is characterized by the implantation and successful adaptation of endometrial tissue in extrapelvic sites. This can include the bowel, lungs, ureters and ovaries with a wide spectrum of severity noted. This chronic condition is thought to affect $7-10 \%$ of women of reproductive age and up to half of all infertile women [1]. Currently, due to the insidious presentations of endometriosis in women of childbearing age it is unsurprising that on average it takes 10.4 years from first symptom to diagnosis and even with the improvements made in imaging modalities this remains a lengthy 8 years. Indeed this can cause several issues including the need for future oophorectomy and hysterectomy as a result of lesion progression [2]. Unfortunately in the majority many women are turned away and their symptoms considered functional in nature many years before this diagnosis is made. This inevitably leads to major disability and impaired quality of life. Indeed due to the nature of this condition many women may mistakenly think the symptoms they suffer are normal [3].

\section{Methods}

Papers were identified by searching medical databases including Pubmed, Cochrane and Google Scholar.

All papers identified were published in peer reviewed journals between 2008-2017. All selected papers were of high quality and dealt with either screening of endometriosis through either adaptation of existing techniques or novel biomarkers.

\section{Results and Discussion}

At present, there are three types of endometriosis recognised; superficial, ovarian and deep infiltrating4. Superficial lesions are likely pelvic in nature and can be identified as blue-black on direct observation. Ovarian endometriosis deposits also known as endometriomas ('chocolate cysts') are psuedocystswhich are much more likely to form adhesions to surrounding structures. Deep infiltrating endometriosis however is defined as extension of endometrial glands $>5 \mathrm{~mm}$ beneath the peritoneal surface and most frequently involves the uterosacral ligaments and is associated with underlying adenomyosis. These lesions are classically related to the cyclical pain patients report and are solid masses of endometrial tissue with associated fibrous and smooth muscle proliferation [4].

The initial symptoms of endometriosis include, dysmenorrhoea, dyspareunia, lower abdominal pain, dysuria and altered bowel habit. Of these dysmenorrhoea is the most common symptom and compounding this up to $20 \%$ of affected women will have other chronic pain conditions including fibromyalgia, migraine and irritable bowel syndrome4.It is due to this varied presentation that clinicians often have to rely on a good history of the clinical signs and symptoms in order to make the diagnosis. One systematic review addressing the usefulness of signs/symptoms in diagnosing endometriosis showed that dysmenorrhoea and dyspareunia are universally present in endometriosis particularly in early stage disease5. Key points within the history include the presence of symptoms and their timing within the women's menstrual cycle, the length of the womens menstrual cycle and the regularity of their periods. However while essential clinical examination of the patient often provides little information with findings such as cervical deviation, cervical tenderness; fixed retroverted uterus or adnexal mass often not found. But when found is more likely to be present when the examination is performed during menstruation [5].

Current understanding is that this condition has both a genetic and environmental components with patients who have an affected first degree relative themselves having 6 times the relative risk of 
developing the condition versus controls [1]. Indeed current opinion is that $51 \%$ of the variation in endometriosis risk is heritable. However, studies looking into the genetic basis of endometriosis have failed to reproduce candidate genes. Recently however Genome Wide Association Studies (GWAS) utilising patients with endometriosis from three continents have shown there to be 10 loci which together only account for $\sim 5 \%$ of underlying endometriosis heritability [6]. As such much more work with regards to DNA methylation and histone modification are required.

Historically, the pathophysiology of endometriosis has been explained by Sampsons theory of retrograde menstruation secondary to fallopian tube reflux, [7]. While this has formed much of the basis of endometrial research and understanding the fact that a paucity of diagnostic techniques for this condition exist remains evident. Indeed even after several decades of research the gold standard remains laparoscopy which incurs great cosmetic, psychological and physical harm but can identify all types of endometriosis [7]. However even this gold standard has potential flaws with visual diagnosis of endometriosis by this process proving to be suboptimal. It is thought that up to half of all positive findings on laparoscopy will be incorrect with differentials including benign inflammatory changes [4].

As laparoscopy represents an extreme method of diagnosing disease given its high frequency within the population, physicians will often initially rely on common and easily accessible imaging modalities such as transvaginal ultrasound (TVUS). TVUS is a common modality used within gynaecology to diagnose a myriad of problems. In a recent systematic review looking at the accuracy of this modality it was found to have a sensitivity of $53 \%, 49 \%$ and $58 \%$ in uterinesacral, rectovaginal septum and vaginal endometriosis respectively [8]. Indeed the problem with TVUS is that often the detail it provides is suboptimal particularly for deep infiltrating endometrial lesions and is not sensitive for large areas of disease [9]. As such MRI is increasingly being used for this reason as well as to diagnose endometriomas, stage disease and confirm the presence of adhesions. This can all be achieved at magnetic field strengths of $\sim 3.0 \mathrm{~T}$. The general consensus at present is that MRI is most sensitive when performed during the first half of the menstrual cycle with a full bladder to remove the anteversion of the uterus. Utilising diffusion weighted imaging alongside apparent diffusion coefficient (ADC) values radiologists have been able to identify with great accuracy endometriomas and solid implants. This is because they demonstrate restricted diffusion patterns versus other pelvic pathologies including haemorrhagic cysts and dermoid cysts [9]. At present most imaging protocols suggest that T1-weighted sequences be used to detect endometrial lesions of $<1 \mathrm{~cm}$ with fat suppression sequences allowing for differentiation of haemorrhagic and cystic lesions10. In comparison pure fibrous lesions will exhibit low T1/T2 imaging intensity while most endometrial lesions exhibit low intensity on T2 imaging. As such the reported specificity of MRI can be as high as 97\% [10].

However as MRI remains a difficult and expensive means of diagnosing such a common condition it remains the hope that a non-invasive test can be made utilising urine, blood, peritoneal fluid etc which with high sensitivity identifies early endometrial disease in the presence of a normal TVUS. Indeed through the development of such a test, it could be sought to prognosticate women based on their levels of said biomarkers. One biomarker which has had a lot of attention is CA-125. This mucinous glycoprotein is expressed by derivatives of coelomic and mullerian epithelium and may be upregulated in both serum and peritoneal fluid in many gynaecological conditions, both benign and malignant in nature where peritoneal inflammation occurs [11]. Early studies of CA125 in this setting has shown that the sensitivity of this marker decreases with more advanced stages of disease and that patients with endometriosis have significantly increased concentrations of CA-125 in the peritoneal fluid versus controls irrespective of the phase of menstruation [12]. A metanalysis of CA-125 performance in this setting revealed that the sensitivity of this marker ranges from $4-100 \%$ with a specificity of $38-100 \%$ [13]. Indeed much like in ovarian cancer where this biomarker is used, it is thought that it may be best utilised when used as part of a biomarker panel, with the sensitivity of this marker improving to $74-94 \%$ with a specificity of 55-75\% when combined with VEGF, Annexin $\mathrm{V}$ and sICAM1 [7]. However, while promising these results need to be ratified according to age and ethnicity of female cohorts, both of which are known to influence CA-125 values. In addition the use of this biomarker remains suboptimal in the diagnosis of endometriosis due to its low specificity. Other serum biomarkers that hold promise in the future include key factors that mediate angiogenesis and include fibroblast growth factor 2 (FGF2) and Endoglin which may also have uses in monitoring progression of endometriosis and its response to treatment [12].

Unlike other tissues of the body the human endometrium is one which in a coordinated manner changes its composition and architecture on a monthly basis. This process is one which sees great cellular proliferation and hormone secretion and eventual hormone withdrawal and apoptosis. Underpinning this process is angiogenesis, which is a key process within endometriosis pathogenesis. In studying this process it has been revealed that microRNAs (miRNAs) are pivotal in this. MicroRNAs are intronic coded genes implicated in regulating gene expression and thus fundamentally control biological systems through gene silencing of several mRNAs simultaneously [14]. Through improvements in next generation sequencing and microarray technology we are now able to study the effects of miRNAs at a genomic scale. Furthermore, the presence of circulating miRNA in the blood has led to research into their application as biomarkers in several conditions including sepsis and myocardial infarction [14]. Early research in this field has revealed differential miRNA expressions between endometrial patients and controls with downregulation of miR-34C-5p, miR-9 and mir-34b, let-7d etc. Let-7d was the first miRNA discovered to promote cellular differentiation with reduced expression of let7 family members well known to be associated with malignancy which has shown to have in endometriosis sensitivity and specificity of $83.3 \%$ and $100 \%$ respectively [14]. Understandably the downregulation of a miRNA leads to a rise of its corresponding 
mRNAtarget gene product it regulates, many of which have been implicated in inflammation, mitosis, cell signalling, angiogenesis and extracellular remodelling.

Currently, miR-125-5P is considered the single most effective diagnostic marker in endometriosis which is upregulated in this setting when measured by quantitative real-time PCR and analysed using receiver operating characteristics. In addition it had the greatest sensitivity and specific of any single miRNA in surgically diagnosed patients. Indeed when combined with miR-451a and mIR-3613-5p a maximum AUC score 1.00 was achieved [15].

A key question remains what causes this dysregulation in the first place. This is because miRNA expression is known to be affected by age, ethnicity, and underlying disease activity. The latter of these is an important point as each of the three types of endometriosis exhibits a different miRNA profile which is affected by the point of the menstrual cycle in which they are measured with a circadian rhythm of expression thought to exist [16]. Due to this and the small study sizes used to date as well as the paucity of establishedmodels in which miRNA research can be conducted in vivo there is much to do before any translations into the clinic can be made [17].

Due to the presence of ectopic endometrial tissue it's of no surprise that there may be an immunological component to this disease. It has been considered that endometriosis may develop as a result of inadequate removal of ectopic tissue due to underlying immune cell alterations including decreased CD36 and LFA-1 expression and decreased T-lymphocyte cytotoxicity [18]. Indeed hormonal alterations in endometriosis are thought to perpetuate this pro-inflammatory state. Moreover, it has been recognised that affected patients demonstrate altered immune responses and often have an increased prevalence of autoimmune disease including systemic lupus erythematosus (SLE). Presently, several autoantibodies have been demonstrated in the serum of endometrial patients including anti- $\alpha$-enolase, anti-PDKI1L, antisyntaxin 5 auto antibodies [19]. Indeed antiendometrial antibody essay has shown to have a positive predictive value of $88 \%$ when used before laparoscopy in the diagnosis of endometriosis [20]. However while measuring the immunological titres is useful, the concern is the fact that these autoantibodies can be found in unaffected women and men. In addition no cut-offs have been assigned to discriminate between active and inactive disease meaning they offer little information or prognostication of affected individuals.

While serum markers remain important, changes to the ultrastructure of the endometrium could offer important insights into early endometrial disease. In recent years there has been considerable interest with regards to nerve biopsy of the endometrium. Over a decade ago it was first noted that endometriosis patients have multiple small unmyelinated Cnerve fibres which can be identified in histological samples of both endometrial lesions and of the endometrium itself through immune histochemistry staining. In contrast in unaffected female patients there are no nerve fibres within the mucosal layers of the endometrium. While this clear distinction sounds perfect for a proposed diagnostic test there have been conflicting reports with some finding that biopsy is neither sensitive or specific with one retrospective study finding that of 29 patients with adenomyosis with no endometriosis at laparoscopy, $69.2 \%$ had positive neuronal immunohistochemistry staining [21,22], However the presence of neuronal fibres is not found routinely pelvic inflammatory disease (PID) or endometrial polyps. Irrespective of this the presence of these new nerve fibres are as of yet unknown however are considered key in the cyclical pain that affected women feel as they are sensitized to endogenous oestrogens during menstruation where at a tissue level inflammation is occurring [22]. Current management of endometriosis involves use of the combined oral contraceptive (COCP) and indeed GnRH agonists confirming that endometriosis is a hormone responsive disease. However, these are plagued with side effects and are not ideal in those women who wish to conceive [4]. It is a result of this that efforts have been made to detect local aromatase activity, which may reflect early stage disease. While this would be a very useful test, early works have identified that aromatase expression in infertile women is no different irrespective of whether endometriosis is the cause. However as there are many steroid regulating proteins which may be implicated, it may be that measuring enzyme levels locally within the endometrium may be a future diagnostic tool as will mapping local oestrogen receptor expression in symptomatic women [23].

\section{Conclusion}

At present endometriosis remains a highly disabling condition that affects a great number of women. While much has been achieved with regards to treatment, diagnosis of this condition remains unchanged from decades past with many women waiting years for a diagnosis. Currently, several new methods of identifying early stage disease have been identified although their significance remains unknown. Furthermore, while much work has been undertaken in identifying biomarkers in endometriosis ,one major problems is few have been sufficiently studied to allow for meta-analysis and thus any conclusions to be made. In order to improve the utility of proposed biomarkers, larger studies must be conducted in patients according to disease subtype and patient presentation to allow for meaningful translation of experimental data to the clinic.

\section{References}

1. Grande G, Vincenzoni F, Milardi D, Pompa G, Ricciardi D, et al. (2017) Cervical mucus proteome in endometriosis. Clin Proteomics 14: 7.

2. Oliveria MAP, Raymundo TS, Soares LC, Pereira TRD, Demôro AVE, et al. (2017) How to use CA-125 more effectively in the diagnosis of deep endometriosis. BioMed Research International 2017: 6.

3. Zhao L, Gu C, Ye M, Zhang Z, Han W, et al. (2017) Identification of global transcriptome abnormalities and potential biomarkers in eutopic endometria of women with endometriosis: A preliminary study. Biomed Rep 6(6): 654-662.

4. Hsu AL, Khachikyan I, Stratton P (2011) Invasive and non-invasive methods for the diagnosis of endometriosis. Clin Obstet Gynecol 53(2): 413-419.

5. Riazi H, Tehranian N, Ziaei S, Mohammadi E, Hajizadeh E, et al. (2015) Clinical diagnosis of pelvic endometriosis: a scoping review. BMC Womens Health 15: 39. 
6. Fassbender A, Burney RO, O DF, D’Hooghe T, Giudice L (2015) Update on biomarkers for the detection of endometriosis. Biomed Res Int 2015: 130854.

7. Guerriero S, Ajossa S, Minguez JA, Jurado M, Mais V, et al. (2015) Accuracy of transvaginal ultrasound for diagnosis of deep endometriosis in uterosacral ligaments, rectovaginal septum, vagina and bladder: systematic review and meta-analysis. Ultrasound Obstet Gynecol 46(5): 534-545.

8. Tadros MY, Keriakos NN (2016) Diffusion MRI versus ultrasound in superficial and deep endometriosis. The Egyptian Journal of Radiology and Nuclear Medicine 47(4): 1765-1771.

9. Bozak AB, Szurowska E, Sawicki S, Liro M (2013) The importance and perspective of magnetic resonance imaging in the evaluation of endometriosis. Biomed Research Int 2013: 436589.

10. Das PM, Bast JR RC (2008) Early detection of ovarian cancer. Biomark Med 2(3): 291-303.

11. Gupta S, Agarwal A, Sekhon L, Krajcir N, Cocuzza M, et al. (2006) Serum and peritoneal abnormalities in endometriosis: potential use as diagnostic markers. Minerva Ginecol 58(6): 527-551.

12. Mol BW, Bayram N, Lijmer JG, Wiegerinck MA, Bongers MY, et al (1998) The performance of CA-125 measurement in the detection of endometriosis: a meta-analysis. Fertil Steril 70(6): 1101-1108.

13. Cho S, Mutlu L, Grechukhina O, Taylor HS (2015) Circulating microRNAs as potential biomarkers for endometriosis. Fertil Steril 103(5): 12521260 .
14. Cosar E, Mamillapalli R, Ersoy GS, Cho S, Seifer B, et al. (2016) Serum microRNAs as diagnostic markers of endometriosis: a comprehensive array based analysis. Fertil Steril 106(2): 402-409.

15. Borghese B, Zondervan KT, Abrao MS, Chapron C, Vaiman D, et al. (2017) Recent insights on the genetics and epigenetics of endometriosis. Clinical Genetics 91(2): 254-264.

16. Teague EM, Print CG, Hull ML (2010) The role of microRNAs in endometriosis and associated reproductive conditions. Hum Reprod Update 16(2): 142-165.

17. Herington JL, Bruner Tran KL, Lucas JA, Osteen KG (2012) Immune interactions in endometriosis. Expert Rev Clin Immunol 7(5): 611-626.

18. Gajbhiye R, Sonawani A, Khan S, Suryawanshi A, Kadam S, et al. (2012) Identification and validation of novel serum markers for early diagnosis of endometriosis. Hum Reprod 27(2): 408-417.

19. Randall GW, Gantt PA, Poe Zeigler RL, Bergmann CA, Noel ME, et al. (2007) Serum antiendometrial antibodies and diagnosis of endometriosis. Am J Reprod Immunol 58(4): 374-382.

20. Zhang X, Lu B, Huang X, Xu H, Zhou C, et al. (2010) Innervation of endometrium and myometrium in women with painful adenomyosis and uterine fibroids. Fertil Steril 94(2): 730-737.

21. Miller EJ, Fraser IS (2015) The importance of pelvic nerve fibres in endometriosis. Women's health 11(5): 611-618.

22. May KE, Villar J, Kirtley S, Kennedy SH, Becker CM (2011) Endometrial alterations in endometriosis: a systematic review of putative biomarkers. Hum Reprod Update 17(5): 637-653. 